Research Article

\title{
C-SIW Rumor Propagation Model with Variable Propagation Rate and Perception Mechanism in Social Networks
}

\author{
Liqing Qiu (i) and Shuqi Liu \\ Shandong Province Key Laboratory of Wisdom Mine Information Technology, College of Computer Science and Engineering, \\ Shandong University of Science and Technology, Qingdao 266590, China
}

Correspondence should be addressed to Liqing Qiu; qiuliqing2019@163.com

Received 21 June 2020; Accepted 6 July 2020; Published 5 August 2020

Guest Editor: Jianxin Li

Copyright (c) 2020 Liqing Qiu and Shuqi Liu. This is an open access article distributed under the Creative Commons Attribution License, which permits unrestricted use, distribution, and reproduction in any medium, provided the original work is properly cited.

\begin{abstract}
The propagation of rumor has become a common phenomenon in social networks. Studying the dynamic propagation of rumor can help locate the key points to control rumor propagation. To further research the internal motivation of state transition, a corrector-ignorant-spreader-weakener (C-SIW) model is proposed in this paper. When the individual changes state to transmit rumor, the neighbor may have a significant impact on rumor propagation. Considering the point, this paper constructs a function to describe the propagation rate, which relates to the state of neighbors and the reputation of the spreader. In addition, perception from life also can cause individual state changes. Based on the above fact, the links from the spreader and the weakener to the corrector are added to describe the perception mechanism. Then, combining the derived average field equations, the steady state of the model is analyzed and verified in experimental simulation. Moreover, the experimental results on different networks show that the perception mechanism reduces the rumor influence. Besides, the variable propagation rate can position the fast-growing stage of rumor propagation more accurately and facilitate the control of rumor propagation.
\end{abstract}

\section{Introduction}

Driven by the rapid development of the Internet and information technology, social networks $[1,2]$ have become an indispensable platform to meet people's information needs and daily communication. With the popularity of social platforms such as WeChat and Facebook, multichannel information enables people to know what is going on quickly and conveniently. However, it is inevitable that some information that is not based on facts, namely, rumors, also can spread rapidly. As a typical social phenomenon, the impact of rumors ranges from confusing the people's minds to endangering public order in various emergencies and crises, which is inestimable. For example, in November 2015, a large amount of information about the "terrorist attack in Baotou" appeared on such platforms as WeChat and Weibo, which has triggered public panic. In this case, it can help to stabilize people's mood that officials and the people on the scene clarify the cause and process of the incident in time.
Shortly afterward, it is verified that the two brothers smashed the passing vehicles in front of the farmer's market after getting drunk, which resulted in serious traffic jam rather than terrorist attack. Then the matter gradually subsides after people get to know the fact. Therefore, a thorough understanding of internal mechanisms and external factors is the most basic issue to help control the spread of rumors faster and more effectively.

With the rumor propagation gradually becoming a normal, the dynamic spread of rumors has become a hot research direction in the social network field. Considering that there are many similarities, the rumor models are mainly based on the epidemic spreading models. The first rumor model, Daley-Kendall (DK) model [3], divided the crowd into three types: one is the people who have not heard the rumor (ignorants), the second is the people who have heard the rumor and spread it (spreaders), and the third is the people who have heard the rumor but did not spread it (stiflers). The Maki-Thompson (MK) model [4], a variant of 
the DK model, assumed that when two spreaders contact each other, the initial spreader will spread rumor no longer and become a stifler. Admittedly, the typical DK and MK rumor models established a solid foundation for future scholars' research. Sudbury [5] studied the influence of the network topology factor, which is not considered in the DK and MK models during propagation process. Afterward, Moreno et al. [6] combined the MK model with epidemics susceptible-infected-recovered (SIR) model and proposed a novel rumor model, in which S, I, and R represent spreaders, ignorants, and stiflers, respectively. Then, some rumor models explored the influence on the propagation process by considering various mechanisms such as forgetting [3, 4], stifling [7], trust [8], hesitating [9], and self-growth [10]. Nevertheless, these models did not consider that the experience from life may also promote individual state transition. Moreover, with the help of immune strategy [11, 12] to control epidemic infection, many researchers applied the idea of immunizing to prevent rumor propagation by spreading the truth. In addition, information diffusion [13] has been extensively studied from a different perspective. Researchers took different factors including geosocial data [14], community [15-17], location prediction [18], and topic search [19] into account, which evaluated the influence propagation in detail. By mapping the spread of rumors with the spread of wildfire in forest, Indu and Thampi [20] discussed an approach that calculated the probability of a node being affected by rumor and modeled the rumor propagation in a network. In the last few years, different from the previous studies, scholars have begun to focus on the perspective of individual. Afassinou [21] introduced the education rate of the population as a factor to examine the evolution of rumor, which proved that the improvement of individuals education rate can promote the termination of rumor propagation. Further, [22, 23] researched individual's awareness and debunking behavior, respectively, which advocated restraining rumor propagation by improving user awareness, imposing punishment on spreaders, and encouraging authoritative influencers to spread the truth. However, they ignored that neighbors have a certain impact on individuals. For example, a large number of stiflers in neighbors may reduce the probability of individual spreading rumor, and the spreader with big reputation may also have a greater impact on propagation.

Through the research studies of the above models considering various mechanisms and factors, more comprehensive understanding and cognition of the rumor propagation can be obtained. In reality, the propagation rate of rumors usually tends to increase with people's attention to it. However, few existing models exhibit this characteristic. They assume that the rumor propagation rate is a constant throughout the whole propagation process, which ignores the potential influence of ignorant's neighbors [24] on the ignorant when the ignorant turns into a spreader. There is also such a social phenomenon, that is, the attitude of individuals towards rumor would change due to the perception from life. Inspired by the above, starting with the individual's attitude to rumors, the stiflers are divided into weakeners and correctors based on the fundamental SIR model. In this paper, a Corrector-Ignorant-SpreaderWeakener (C-SIW) rumor propagation model considering the variable propagation rate and perception mechanism is proposed. In particular, spreaders, weakeners, and correctors are defined as forwarding propagation, neutral propagation, and negative propagation state, respectively. In detail, the main research contributions of the paper can be reflected in the following aspects:

(i) Considering the impact of social relations, the rumor propagation rate of C-SIW model depends on the comprehensive influence of neighbors and the reputation of the spreader when the ignorant is infected by a spreader.

(ii) Considering the possible situations in life, the paper adds a link from the spreader and the weakener to the corrector triggered by perception mechanism, respectively. Besides, the perception mechanism from the weakener to the corrector breaks the dynamic balance between the two states.

(iii) The C-SIW model is simulated on the generated BA and WS networks, and the experimental results are compared with those of the real social network Facebook.

The rest of the paper is organized as follows. The next section details the proposed C-SIW model and related mechanism. The third section carries on the steady state analysis to the model. The experimental simulation is generated in Section 4, and the results are explained systematically. Further, the last section covers the conclusions and future work.

\section{C-SIW Rumor Propagation Model with Variable Propagation Rate and Perception Mechanism}

In this section, the fundamental SIR model and the C-SIW model are presented in detail. Uniformly, the research environment social network of the model can be represented as an undirected graph $G(V, E)$ containing $N$ individuals. All the individuals in social networks act as nodes set $V$, and different-connected edges set $E$ contains the association between different individuals.

2.1. SIR Model. Up to now, the epidemics SIR model has made a great contribution to the development of rumor propagation. Before introducing the proposed C-SIW model, in this subsection, the basic SIR model is illustrated firstly.

In the SIR model, the individuals in the system can be divided into three categories: susceptible (the healthy individuals who may be infected, denoted as S), infected (the individuals who are in a diseased state and are contagious, denoted as I), and recovered (the individuals who recover and gain immunity or die after infection, denoted as R). The specific infection process is shown in Figure 1. The susceptible may contact an infected and then converts to an 


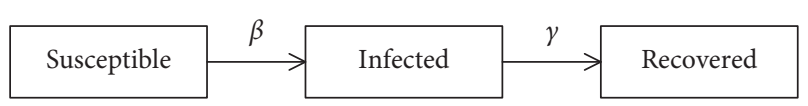

FIgURE 1: The infection process of the SIR model.

infected with the probability of $\beta$; and the recovered comes from the infected people who recovered from disease or died with the probability of $\gamma$.

Then, to describe the above infection rules, the average field equations can be established as follows:

$$
\begin{aligned}
\frac{\mathrm{d} S(t)}{\mathrm{d} t} & =-\beta S(t) I(t), \\
\frac{\mathrm{d} I(t)}{\mathrm{d} t} & =\beta S(t) I(t)-\gamma I(t), \\
\frac{\mathrm{d} R(t)}{\mathrm{d} t} & =\gamma I(t) .
\end{aligned}
$$

In equation (1), $S(t), I(t)$, and $R(t)$ represent the density of the susceptible, the infected, and the recovered at time $t$, respectively; and, in the system, $S(t)+I(t)+R(t)=1$ is maintained at any time.

\subsection{Evolution Process of the C-SIW Rumor Propagation} Model. To further study the intrinsic conversion motivation of promoting rumor propagation, the C-SIW model also follows the footsteps of predecessors and extends with the SIR as the fundamental model. In the C-SIW model, according to the different effects of each individual on rumor spreading, each individual in the system is in one of the following states at time $t$ : the ignorant (who has not heard the rumor yet, similar to the susceptible), the spreader (who has heard the rumor and spread it, similar to the infected), and the stifler (similar to the recovered) that can be subdivided into the weakener (who has heard the rumor and no longer spread it because he/she is not interested or unsure about the truth of the rumor) and the corrector (who can distinguish the rumor and suppress the rumor spreading by disseminating the truth after he/she has heard the rumor). Different from the previous researches, in the process of rumor propagation, the state of the weakener may spontaneously transform into the corrector due to the effect of perception mechanism, rather than not into another state. The total propagation process is carried out through the link edges between the nodes. Specifically, the evolution process of the C-SIW rumor model is shown in Figure 2.

Combined with the state transition of the nodes in Figure 2, the rules for rumor propagation of the C-SIW model are as follows:

(1) When the spreader in the system contacts an ignorant, there are three state transition options for the ignorant. One is that no matter the ignorant will believe or doubt the rumor spread by the spreader, he/she will spread the rumor with propagation rate $\beta$. The second is that the ignorant has rich

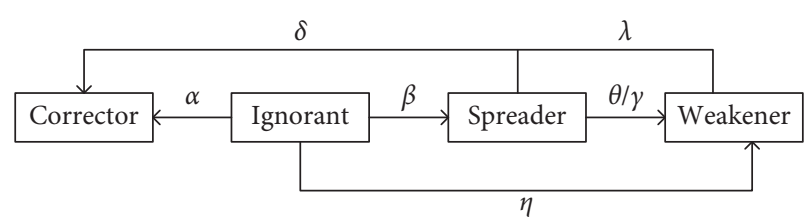

FIGURE 2: The evolution process of the C-SIW rumor propagation model.

knowledge to recognize the truth of the rumor and spread the truth with a containment rate of $\alpha$ to prevent the friends from being cheated, that is to say, converts to a corrector. The last possibility is that the ignorant is not interested in rumor or does not know whether the rumor is true or false, which shows a negative attitude and he/she turns into a weakener with the rate of $\eta$. Here, suppose that $\alpha+\beta+\eta=1$.

(2) With the contact between individuals, the spreader may convert to a weakener with the stifling rate $\gamma$; or, after being influenced by the weakener and the corrector, the spreader doubts his cognition and converts to a weakener with the immune rate $\theta$. Another situation is that the spreader himself/herself is conscious of the inaccuracy of the rumor by the perception of life and then converts to a corrector with a certain probability, called spontaneous control rate $\delta$.

(3) Similarly, due to the perception from life experience, the weakener may realize that the rumor is misleading. In this case, the weakener's attitude towards life, which is in the state of neutral propagation at this time, transforms into a positive way by spreading the fact with the probability of $\lambda$, named fact transmission rate.

2.3. Average Field Equations. Based on the propagation rules above, the average field equations of the C-SIW model are established to describe the propagation process. First, the proportions of ignorant, spreader, weakener, and corrector in the whole system at time $t$, that is, the density value, are marked as $I(t), S(t), W(t)$, and $C(t)$, respectively. The average degree of the network is expressed by $k$ in this paper. Consistent with most of the former researches, the states in the system follow the following normalization conditions:

$$
I(t)+S(t)+W(t)+C(t)=1
$$

Then, the average field equations of C-SIW model are shown as the following equations:

$$
\begin{aligned}
\frac{\mathrm{d} I(t)}{\mathrm{d} t} & =-k \beta S(t) I(t)-k \alpha S(t) I(t)-k \eta S(t) I(t) \\
\frac{\mathrm{d} S(t)}{\mathrm{d} t} & =k \beta S(t) I(t)-\gamma S(t)-\delta S(t)-k \theta S(t)(W(t)+C(t)),
\end{aligned}
$$




$$
\frac{\mathrm{d} W(t)}{\mathrm{d} t}=k \eta S(t) I(t)+\gamma S(t)-\lambda W(t)+k \theta S(t)(W(t)+C(t))
$$

$$
\frac{\mathrm{d} C(t)}{\mathrm{d} t}=k \alpha S(t) I(t)+\delta S(t)+\lambda W(t)
$$

Here, in order to satisfy the generality, there is only one randomly selected individual spreading the rumor when $t=0$. That is, the initial conditions for the evolution of the model are set as follows:

$$
\begin{aligned}
& I(0)=\frac{N-1}{N} \approx 1, \\
& S(0)=\frac{1}{N} \approx 0, \\
& W(0)=0, \\
& C(0)=0 .
\end{aligned}
$$

2.4. Propagation Mechanisms. After understanding the propagation process of the model, the propagation mechanisms are introduced to explore the potential motives for influencing the rumor propagation. In the subsection, the variable propagation rate and perception mechanism are explained in detail.

2.4.1. Variable Propagation Rate. To more closely express that the propagation rate is not always constant, the paper introduces a function to describe this characteristic. As far as we know, the spread of rumors often depends on social relations, which are a strong feature performed by social networks. Therefore, particular research on the role of social relations in rumor propagation is conducted. As supposed in Section 1, both weakener and corrector are negative parties for rumor propagation. In such a situation, this paper considers that when an individual is infected to spread the rumor, social relationship is a comprehensive effect of potential contact between individual and neighbors. Meanwhile, the reputation of the spreader is also a critical factor contributing to propagation. The greater the reputation of the spreader is, the easier it is to gain individual trust in rumor. That is to say, the propagation probability of each individual is different by considering their situation. Therefore, for each individual $i$, such a function to describe the rumor propagation rate $\beta$ concretely at time $t$ can be defined as follows:

$$
\beta_{i}(t)=\beta_{0} e^{-\left(\left(\left(\left(C_{i}(t)+W_{i}(t)-S_{i}(t)\right) / \operatorname{tot}_{i}(t)\right)+(1-f)\right) / 2\right)},
$$

where $\beta_{0}$ represents the initial propagation rate; $\left(\left(C_{i}(t)+\right.\right.$ $\left.\left.W_{i}(t)-S_{i}(t)\right) / \operatorname{tot}_{i}(t)\right)$ is assumed as the comprehensive influence of neighbors on individual $i ; C_{i}(t), W_{i}(t)$, and $S_{i}(t)$ denote the number of correctors, weakeners, and spreaders in the total neighbors tot ${ }_{i}(t)$ of individual $i$ at time $t$, respectively; $f$ reflects the reputation of the spreader; $e$ means that the function is based on the irrational number of 2.7182818 ... For example, there is a social network $G$ as shown in Figure 3. Assume that nodes $2,4,7$, and 9 are ignorant, and nodes 1 and 5 are spreaders. Similarly, nodes 6 and 8 belong to the correctors, and node 3 is a weakener. For node 2, the neighbors' influence on it can be expressed as $\left(\left(C_{i}(t)+W_{i}(t)-S_{i}(t)\right) /\right.$ tot $\left._{i}(t)\right)=$ $((1+1-1) / 4)=0.25$. Therefore, assuming that $f=0.6$ and $\beta_{0}=0.7, \beta_{\text {node } 2}=0.7^{*} e^{-((0.25+(1-0.6)) / 2)} \approx 0.5317$ when node 2 is infected by node 5 to spread rumor.

2.4.2. Perception Mechanism. In actual life, people are always impressed by the fact they have experienced, which makes it easy to get them against the rumor. To prevent more people from being deceived by rumor, they spread the fact with strong motivation. The above is the source of perception mechanism and the perception mechanism will take place on the spreader and weakener in the C-SIW rumor propagation system. This is reflected in the spontaneous control rate $\delta$ and fact transmission rate $\lambda$, respectively, when the spreader and weakener convert to the corrector as described in Section 2.2. To be specific, the parameters spontaneous control rate $\delta$ and fact transmission rate $\lambda$ are both assumed to be an adjustable constant between 0 and 1 in this paper.

The above provides a general description of the proposed C-SIW model. Since the obtained average field equations (i.e., equations (3)-(6)) describe the propagation rules of the C-SIW model, the next work is to analyze systematically the dynamic propagation of rumor based on the average field equations.

\section{Steady-State Analysis}

As time goes on, the influence of rumor will have a range, which is the final size of the rumor. In a common way, the paper uses an indicator $R$ to measure it, that is, $R=\lim _{t \longrightarrow \infty} R(t)=R(\infty)$. There are $I=\lim _{t \longrightarrow \infty} I(t)=$ $I(\infty), S=\lim _{t \longrightarrow \infty} S(t)=S(\infty)=0, W=\lim _{t \longrightarrow \infty} W(t)=$ $W(\infty)$, and $C=\lim _{t \rightarrow \infty} C(t)=C(\infty)$ when $t \longrightarrow \infty$. In addition, it is pointed out that the weakeners and the correctors are the components of the stiflers in Section 1. Therefore, $R=R(\infty)=W(\infty)+C(\infty)=W+C=1-I-$ $S=1-I$. Here, the final influence range $R$ of rumor is researched by making use of the average field equations. First, dividing the sum of equations (5) and (6) by equation (3), there is 


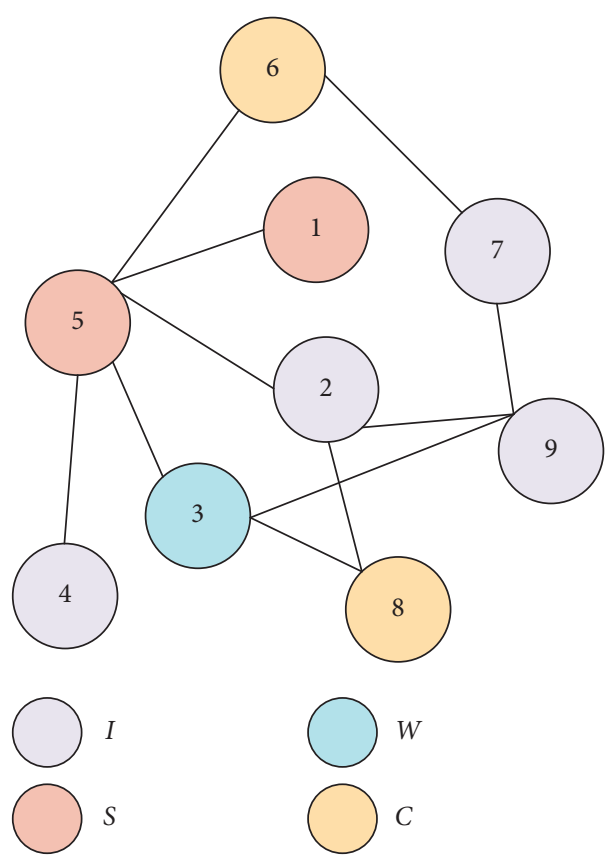

FIgURE 3: A social network $G$.

$$
\begin{aligned}
\frac{\mathrm{d} R(t)}{\mathrm{d} I(t)} & =\frac{\mathrm{d}(W(t)+C(t))}{\mathrm{d} I(t)} \\
& =\frac{\mathrm{d} W(t)+\mathrm{d} C(t)}{\mathrm{d} I(t)} \\
& =\frac{k \eta S(t) I(t)+\gamma S(t)-\lambda W(t)+k \theta S(t)(W(t)+C(t))+k \alpha S(t) I(t)+\delta S(t)+\lambda W(t)}{-k(\beta+\alpha+\eta) S(t) I(t)} \\
& =\frac{k(\alpha+\eta) S(t) I(t)+(\gamma+\delta) S(t)+k \theta S(t)(W(t)+C(t))}{-k S(t) I(t)} \\
& =-(\alpha+\eta)-\frac{\gamma+\delta}{k I(t)}-\frac{\theta R(t)}{I(t)} .
\end{aligned}
$$

Assuming that $R(t)=y$ and $I(t)=x,(\mathrm{~d} R(t) / \mathrm{d} I(t))=$ $-(\alpha+\eta)-((\gamma+\delta) / k I(t))-(\theta R(t) / I(t))$ can be expressed as $\quad(\mathrm{d} y / \mathrm{d} x)=-(\alpha+\eta)-((\gamma+\delta) / k x)-(\theta y / x)$; i.e., $(\mathrm{d} y / \mathrm{d} x)+(\theta / x) y=-((\gamma+\delta) / k x)-(\alpha+\eta)$. According to the solution of the first-order linear nonhomogeneous equation, it can be obtained that

$$
\begin{aligned}
y & =e^{-\int(\theta / x) \mathrm{d} x}\left[\int\left(-\frac{\gamma+\delta}{k x}-(\alpha+\eta)\right) e^{\int(\theta / x) \mathrm{d} x} \mathrm{~d} x+C_{1}\right] \\
& =e^{-\theta \ln x}\left[\int\left(-\frac{\gamma+\delta}{k x}-(\alpha+\eta)\right) e^{\theta \ln x} \mathrm{~d} x+C_{1}\right] \\
& =x^{-\theta}\left[\int\left(-\frac{\gamma+\delta}{k x}-(\alpha+\eta)\right) x^{\theta} \mathrm{d} x+C_{1}\right] \\
& =x^{-\theta}\left[-\frac{\gamma+\delta}{k \theta} x^{\theta}-\frac{\alpha+\eta}{\theta+1} x^{\theta+1}+C_{1}\right]
\end{aligned}
$$

$$
=-\frac{\gamma+\delta}{k \theta}-\frac{\alpha+\eta}{\theta+1} x+C_{1} x^{-\theta} .
$$

Therefore,

$$
R(t)=-\frac{\gamma+\delta}{k \theta}-\frac{\alpha+\eta}{\theta+1} I(t)+C_{1} I(t)^{-\theta} .
$$

Theorem 1. There exists propagation threshold $\beta_{c}=((\gamma+$ $\delta) / k$ ) in the C-SIW model, and only when $\beta>\beta_{c}$ can the rumor be spread.

Proof. Taking the initial condition equation (7) into equation (11), it can be obtained that $R(0)=-((\gamma+\delta) / k \theta)-$ $((\alpha+\eta) /(\theta+1)) I(0)+C_{1} I(0)^{-\theta}$. After that, by solving the above equation, $C_{1}=((\gamma+\delta) / k \theta)+((\alpha+\eta) /(\theta+1))$. At this point, attention should be paid to the fact that all parameters are between 0 and 1 in the proposed model and it is easy to know that $C_{1}>0$. Further, by analyzing equation (11), we can get that when $t \longrightarrow \infty$, 


$$
\begin{aligned}
R & =R(\infty) \\
& =-\frac{\gamma+\delta}{k \theta}-\frac{\alpha+\eta}{\theta+1} I(\infty)+C_{1} I(\infty)^{-\theta} \\
& =-\frac{\gamma+\delta}{k \theta}-\frac{\alpha+\eta}{\theta+1} I+C_{1} I^{-\theta}
\end{aligned}
$$

Taking $I=1-R$ from $R=1-I$ transformation into equation (12), we can get

$$
R=-\frac{\gamma+\delta}{k \theta}-\frac{\alpha+\eta}{\theta+1}(1-R)+C_{1}(1-R)^{-\theta}
$$

Arrange equation (13) and assume that

$$
f(R)=-\frac{\alpha+\eta}{\theta+1}(1-R)-R+C_{1}(1-R)^{-\theta}-\frac{\gamma+\delta}{k \theta} .
$$

Then, the final size of rumor is analyzed by the properties of $R$ in the domain interval $(0,1)$. The derivation of function $f(R)$ on $R$ is $f^{\prime}(R)=((\alpha+\eta) /(\theta+1))-1+C_{1} \theta(1-R)^{-\theta-1}$ and $f^{\prime \prime}(R)=C_{1} \theta(\theta+1)(1-R)^{-\theta-2}$. Because of $C_{1}>0$, it is obvious that $f^{\prime \prime}(R)>0$. Therefore, $f(R)$ is a concave function. Besides, according to equation (14), there are $f(0)=0$ and $\quad \lim _{R \longrightarrow 1^{-}} f(R)=C_{1}\left(1-1^{-}\right)^{-\theta}-((\gamma+\delta) /$ $k \theta)-1=\infty$. Under the circumstance, if $f(R)$ has another nonzero root $R^{*}\left(0<R^{*}<1\right)$, it needs to satisfy that $f^{\prime}(0)=$ $((\alpha+\eta) /(\theta+1))-1+C_{1} \theta=((\alpha+\eta) /(\theta+1))-1+(((\gamma+$ $\delta) / k \theta)+((\alpha+\eta) /(\theta+1))) \theta<0$; that is, $\beta>((\gamma+\delta) / k)$. At the moment, there is a propagation threshold $\beta_{c}=((\gamma+\delta) / k)$. It is necessary to notice the condition that the propagation probability $\beta>\beta_{c}$ must be met, so as to make the rumor spread.

Recently, the strategy has been introduced to inhibit the spread of rumors by immunizing some individuals. Here, at the beginning of spreading, the individuals with the proportion of $p$ in the total individuals are randomly immunized, which means that these individuals will not spread rumor and will inhibit rumor spreading. In this case, the initial conditions for rumor propagation are

$$
\begin{aligned}
I(0) & =1-p, \\
S(0) & =0, \\
W(0) & =0, \\
C(0) & =p .
\end{aligned}
$$

Theorem 2. Considering the implementation of immune strategy for the rumor model, the C-SIW model has an immune proportion threshold $p_{c}=((k \beta-\gamma-\delta) / k(\theta+\beta))$. On the basis of satisfying $\beta>\beta_{c}$ (i.e., $\left.\beta_{c}=((\gamma+\delta) / k)\right)$, only when $p<p_{c}$ can the rumor be spread.

Proof. Bringing the initial conditions in equation (15) into equation (11), there is

$$
\begin{aligned}
R(0) & =-\frac{\gamma+\delta}{k \theta}-\frac{\alpha+\eta}{\theta+1} I(0)+C_{2} I(0)^{-\theta} \\
& =-\frac{\gamma+\delta}{k \theta}-\frac{\alpha+\eta}{\theta+1}(1-p)+C_{2}(1-p)^{-\theta} \\
& =W(0)+C(0) \\
& =p
\end{aligned}
$$

From equation (16), $C_{2}=p(1-p)^{\theta}+((\gamma+\delta) / k \theta)$ $(1-p)^{\theta}+((\alpha+\eta) /(\theta+1))(1-p)^{\theta+1}$. It can be observed that $C_{2}>0$. Furthermore,

$$
\begin{aligned}
R & =R(\infty) \\
& =-\frac{\gamma+\delta}{k \theta}-\frac{\alpha+\eta}{\theta+1} I(\infty)+C_{2} I(\infty)^{-\theta} \\
& =-\frac{\gamma+\delta}{k \theta}-\frac{\alpha+\eta}{\theta+1} I+C_{2} I^{-\theta} \\
& =-\frac{\gamma+\delta}{k \theta}-\frac{\alpha+\eta}{\theta+1}(1-R)+C_{2}(1-R)^{-\theta}
\end{aligned}
$$

Assume that $g(R)=-((\alpha+\eta) /(\theta+1))(1-R)-R+$ $C_{1}(1-R)^{-\theta}-((\gamma+\delta) / k \theta)$. Similarly, by deriving $g(R)$, there are $g^{\prime}(R)=((\alpha+\eta) /(\theta+1))-1+C_{2} \theta(1-R)^{-\theta-1}$ and $g^{\prime \prime}(R)=C_{2} \theta(\theta+1)(1-R)^{-\theta-2}>0$. That is, $g(R)$ is a concave function. $g(p)=-((\alpha+\eta) /(\theta+1))(1-p)-p+$ $C_{2}(1-p)^{-\theta}-((\gamma+\delta) / k \theta)=0 \quad$ and $\quad \lim _{R \rightarrow 1^{-}} g(R)=$ $C_{2}\left(1-1^{-}\right)^{-\theta}-((\gamma+\delta) / k \theta)-1=\infty$. Then, under the constraint of $g^{\prime}(p)<0$ (i.e., $\left.p<((k \beta-\gamma-\delta) / k(\theta+\beta))\right)$, $g(R)$ will have two roots $p$ and $R^{*}\left(p<R^{*}<1\right)$. As a result, it can be concluded that there exists an immune proportion threshold $p_{c}=((k \beta-\gamma-\delta) / k(\theta+\beta))$ in the model, and the rumor can spread when immune proportion $p<p_{c}$.

\section{Simulation Results and Analysis}

In the section, utilizing the popular WS network [25] and BA network [26], the paper simulates and analyzes the steady state of the model. Furthermore, considering that the real social network has more complex topological structure and clustering characteristics than the generated network, the characteristics of the model in the real-world network are worth exploring. As a social platform, Facebook is closer to people's lives. Therefore, the paper investigates the dynamic spread of rumors and makes a concrete comparison with the results of the generated WS network and BA network through the experiment on the real social network Facebook. In detail, the employed Facebook network contains 4039 users and 88234 edges [27]. For the WS network and BA network, the number of individuals $N=10^{6}$ is generated to avoid particularities. Besides, the random reconnection probability $p^{\prime}$ and average degree $k$ of the BA network are 0.1 and 6 , respectively, and the average degree $k$ of the WS network is also set to 6 . All the presented results are the average results of 70-time simulations by randomly selecting different initial spreader.

4.1. Verification of the Model's Steady State. First of all, experimental simulation is conducted to discuss the preconditions of rumor propagation. At the same time, the theorems outlined in Section 3 are verified through the analysis of system's steady state.

Figures 4(a) and 4(b) reflect the change of rumor final size $R$ with $\beta$ on WS network and BA network, respectively. Since the rumor propagation rate considered by this paper 


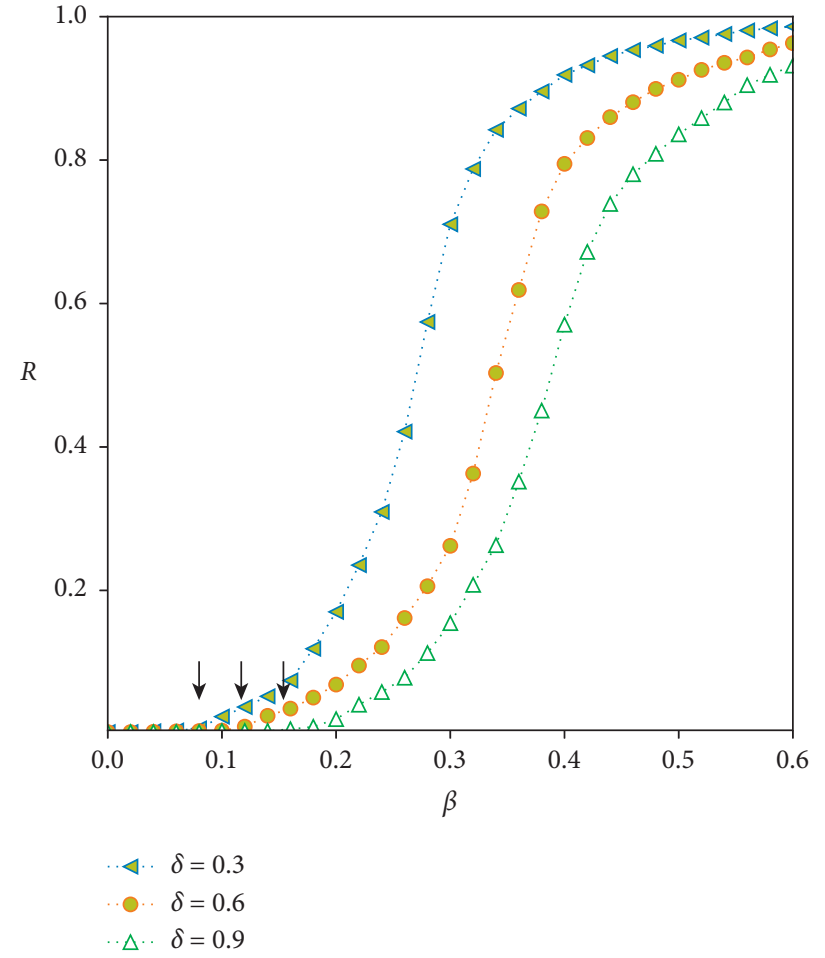

(a)

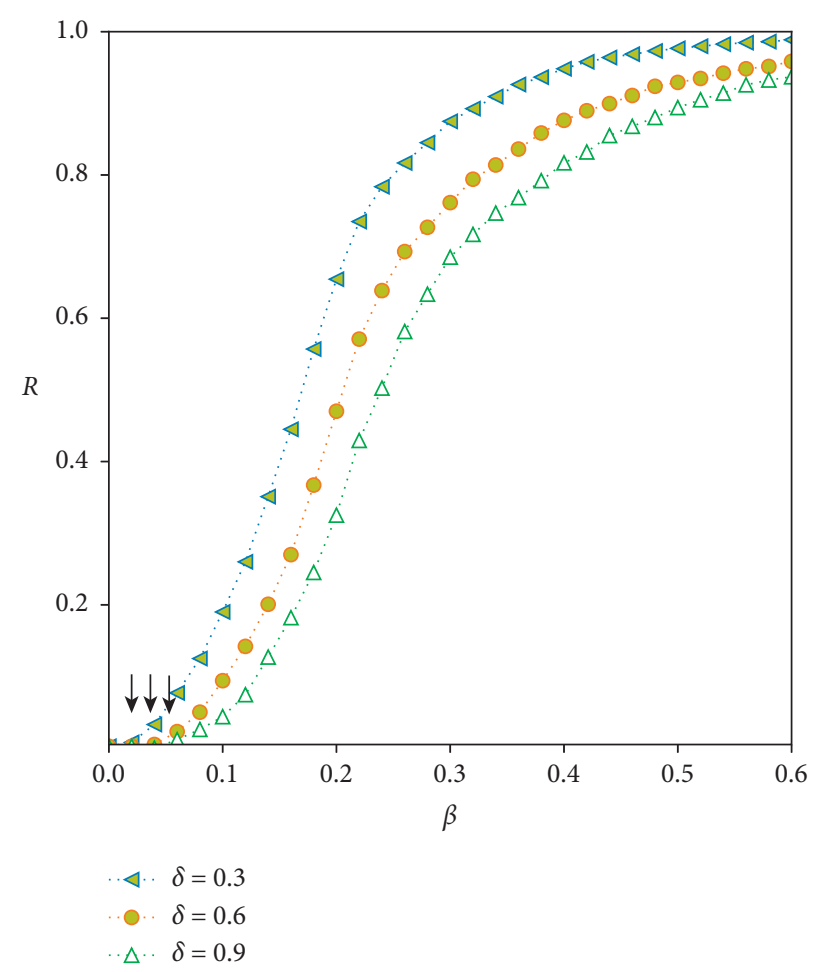

(b)

FIgURE 4: The relationship between the final size $R$ and the propagation rate $\beta$ under different $\delta$ on (a) WS network and (b) BA network. The parameters are $\theta=0.1, \alpha=\eta, r=0.1$, and $\lambda=0.05$.

fluctuates slightly with time, the rumor propagation rate was taken as the average value of all values in the whole system here. From Figure 4(a), we can make out the different propagation threshold according to the different $\delta$ settings through the position indicated by arrows, which is close to $\beta_{c}$ mentioned in Theorem 1. However, the obvious difference is that the propagation threshold in Figure 4(b) appears earlier, which is caused by the hub node in BA network promoting the spread of rumor. Besides, the reason leads to the increase of $R$ being faster than that on WS network with the increase of $\beta$. Furthermore, in both BA network and WS network, the position of the propagation threshold shifts backward and $R$ reduces with the increase of $\delta$. It is caused by the perception mechanism introduced, which is explained further in the next section.

Figure 5 illustrates the trend of the final size $R$ of rumor and its components $C$ and $W$ with different immune proportions $p$ of nodes. Comparing Figures 5(a) and 5(b), it is clear that the immune proportion threshold appears earlier in the WS network than in the BA network. Increasing the number of immune nodes is more convenient to control the propagation of rumor in the WS network, which can be seen from the decline range of $R$ in the same time interval. The main reason is that the degree of nodes in the WS network is more average, while most nodes in the BA network have smaller degree. From the perspective of $C$, it is worth noting that, in the WS network, the trend of $C$ decreases slowly and then rapidly, indicating that the improvement of immune proportion can have an ideal effect. However, for the BA network, the trend realizes that the rumor control reaches a transition from slow to fast as $p$ increases, not as ideal as in the WS network. As the number of the correctors increases, the probability of the spreaders touching the correctors and then converting to the weakeners or correctors is increased, which achieves that individuals will remain uninfected when rumor disappears. In particular, after the immune proportion threshold, $W$ researches zero while $C$ maintains linear growth, which means that there are no weakeners in the system when the rumor disappears. In general, from the simulation on the BA network, Theorem 2 derived is also feasible. In order to prevent people from being hurt by the similar rumors that happened before, relevant staff can sort out relevant data and inform people in advance of what may happen when an event occurs, which can help achieve the effect of early immunization.

4.2. Simulation of Model-Related Mechanisms. Due to the gap between the generated network and the real social network, the differences in the results obtained in different networks cannot be ignored. From this point of departure, the dynamic propagation process of the proposed model on WS network, BA network, and Facebook social network is further discussed by adjusting parameters.

4.2.1. Influence of Variable Propagation Rate on Propagation Process. Figures 6(a)-6(d) demonstrate the effect of neighbors' influence and spreader's reputation on rumor 


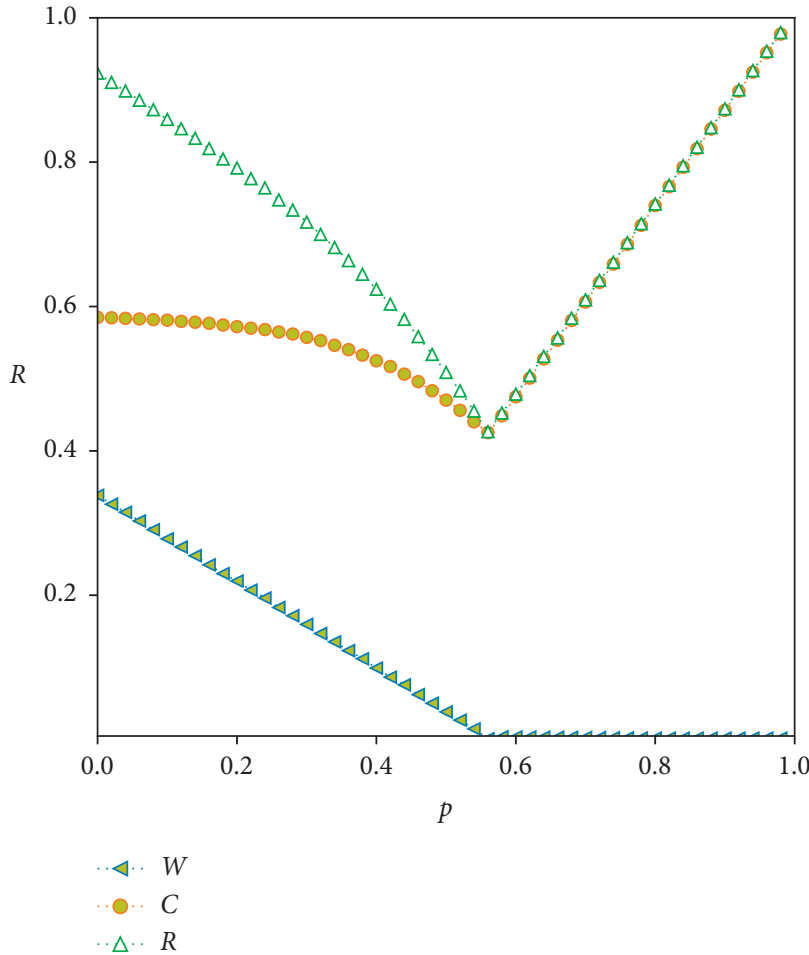

(a)

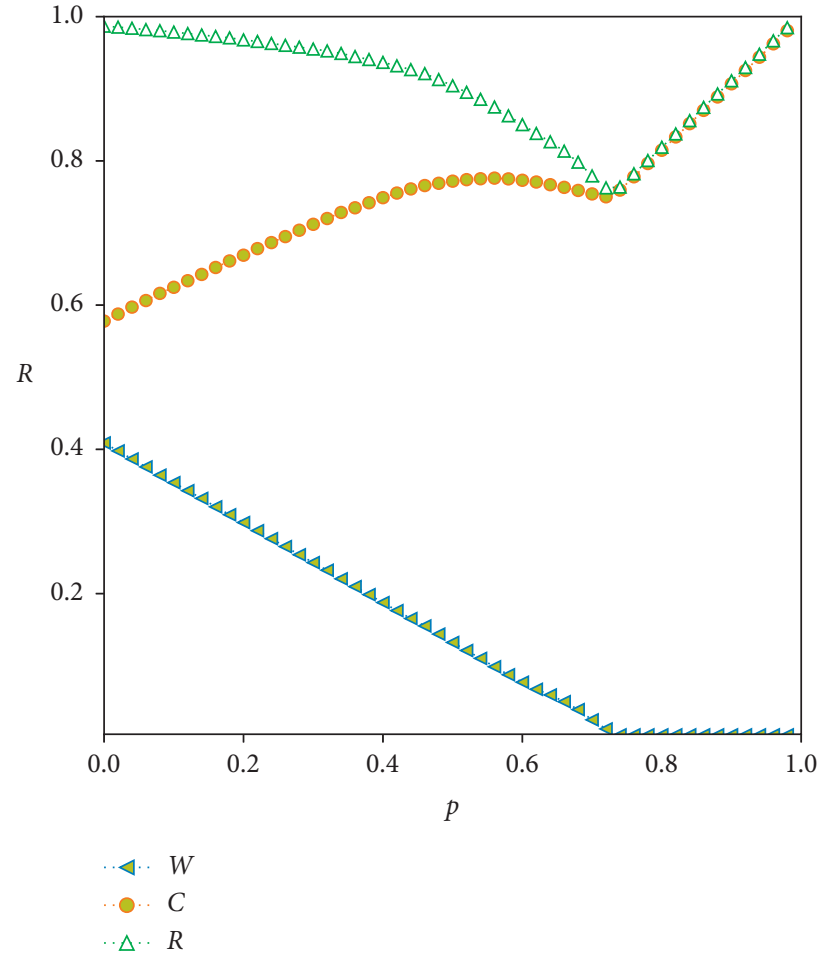

(b)

Figure 5: The relationship between the final size $R$ and the immunization percentage $p$ on (a) WS network and (b) BA network. The parameters are $\alpha=0.2, \beta_{0}=0.6, f=0.7, \eta=1-\beta-\alpha, \theta=0.1, r=0.1, \delta=0.2$, and $\lambda=0.05$.

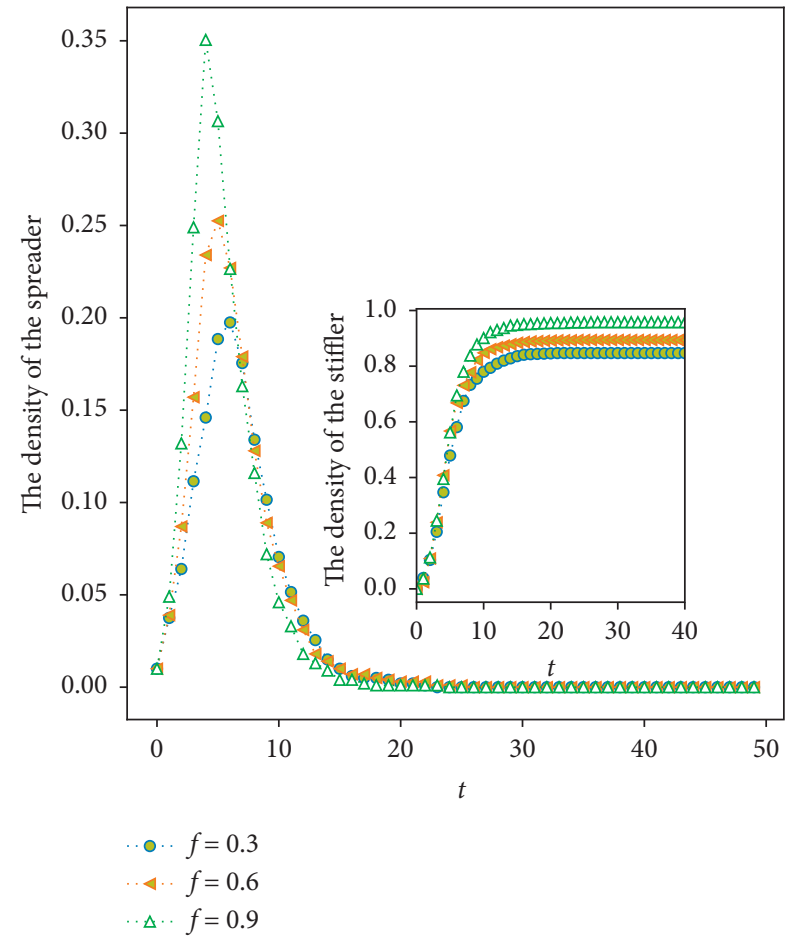

(a)

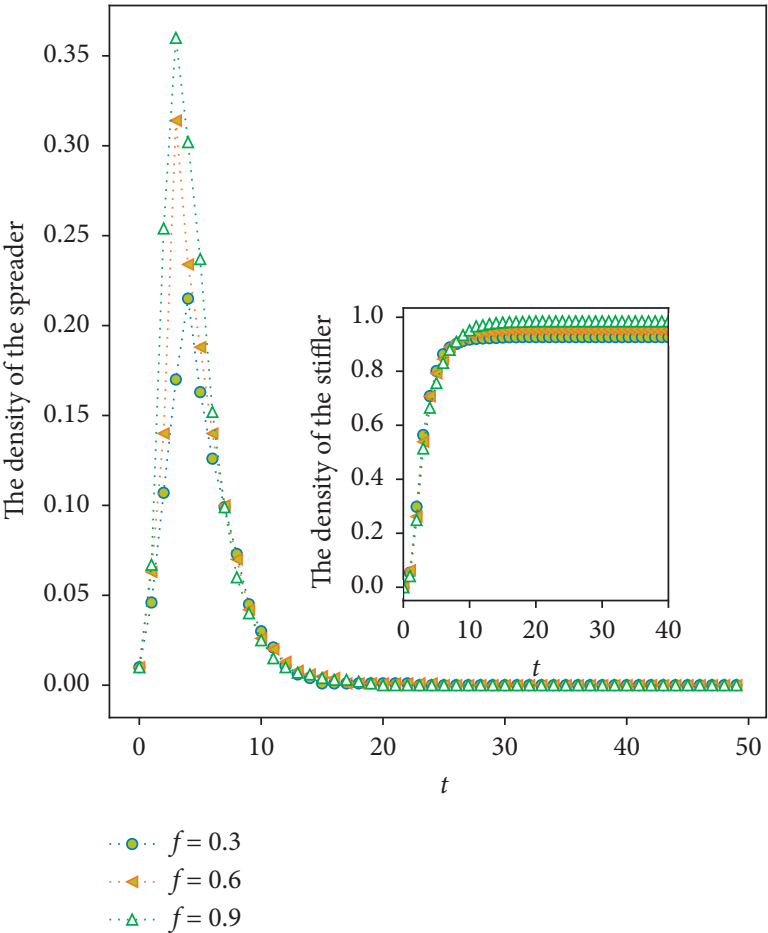

(b)

Figure 6: Continued. 


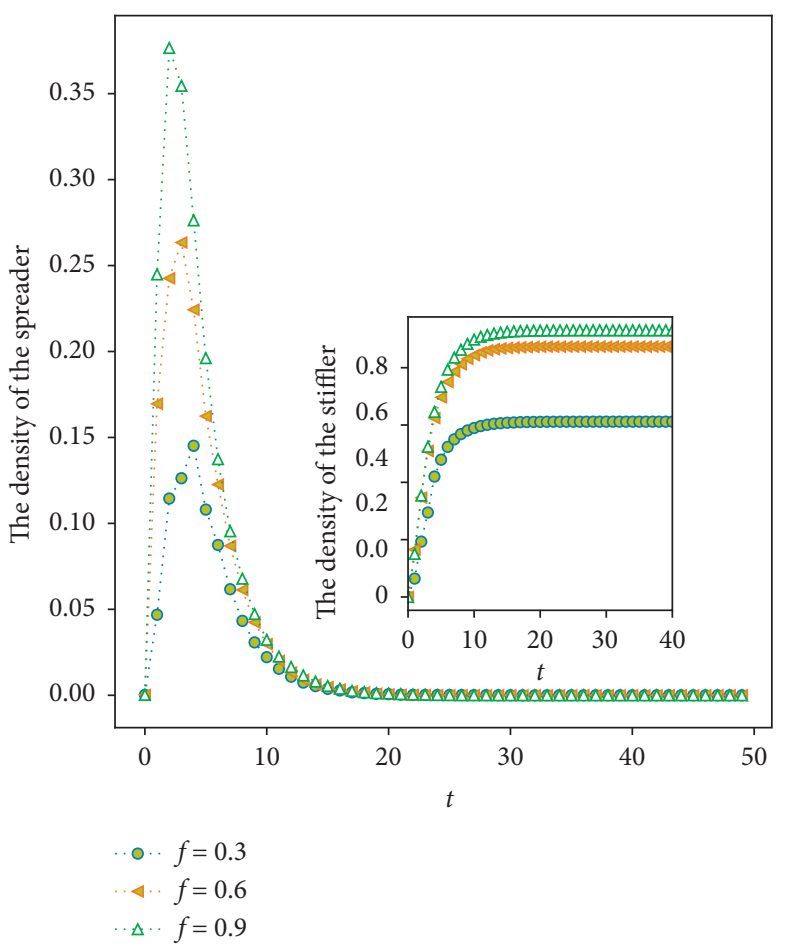

(c)

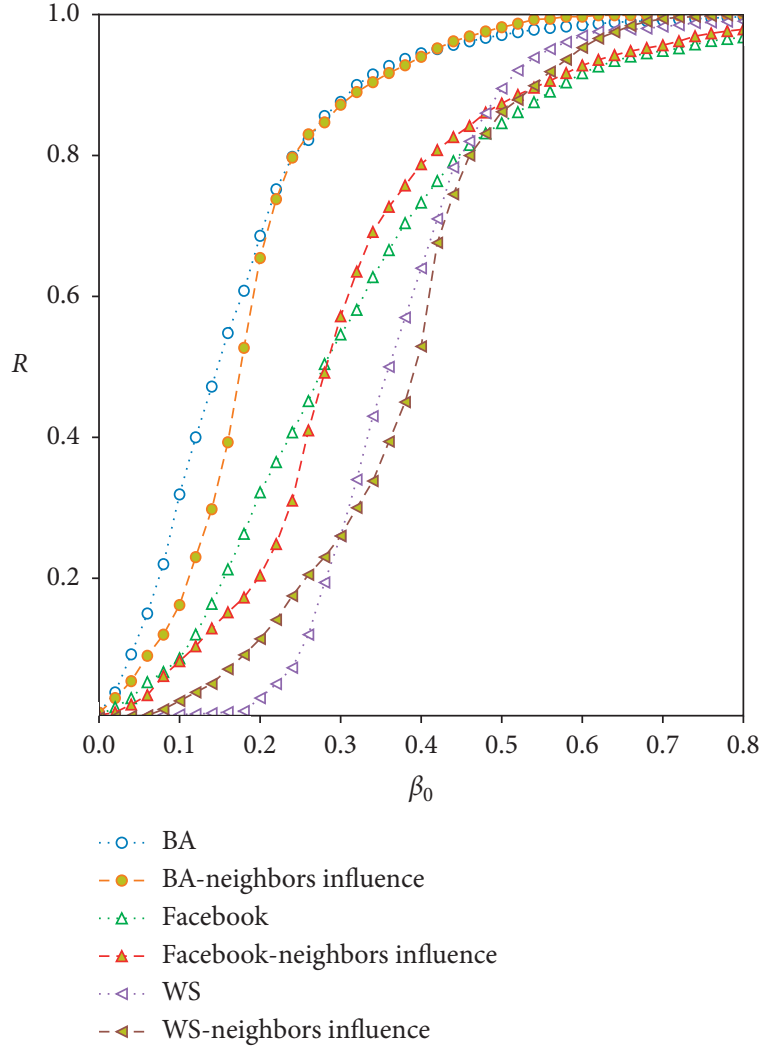

(d)

FIgURE 6: The density trend of state with different $\beta$. The density of spreader and stifler with different $f$ on (a) WS network, (b) BA network, and (c) Facebook. (d) The relationship between the final size $R$ and the initial propagation rate $\beta_{0}$ with and without the comprehensive influence of neighbors on WS, BA, and Facebook networks.

propagation in the variable propagation rate function $\beta_{i}(t)=\beta_{0} e^{-\left(\left(\left(\left(C_{i}(t)+W_{i}(t)-S_{i}(t)\right) / \operatorname{tot}_{i}(t)\right)+(1-f)\right) / 2\right)}$.

First of all, Figures 6(a)-6(c) describe, respectively, the role played by the different reputations of the spreader in WS, BA, and Facebook network, where the reputation of the spreader is assumed to be $0.3,0.6$, and 0.9 with the same interval. The parameters are $\alpha=0.2, \beta_{0}=0.6, \eta=1-\beta-\alpha$, $\theta=0.1, r=0.1, \delta=0.2$, and $\lambda=0.05$. As shown in the figures, the peak density of the spreader increases with the increase of the reputation of the spreader, and the corresponding final rumor size also increases, which shows the famous celebrity effect [28]. The higher the reputation is, the more trust the spreader can gain by spreading rumor, thus promoting the spread of rumor. Inspired by the experiment, people with high reputation should improve their ability of discrimination and exert their influence in suppressing the spread of rumor.

Then, Figure 6(d) displays a comparison of neighbors comprehensive influence on the individual in each network with the change of initial propagation rate $\beta_{0}$, in which the measure is the final rumor size $R$. The parameters are $f=0.6$, $\alpha=\eta, \theta=0.1, r=0.1, \delta=0.2$, and $\lambda=0.05$. Independently, a common feature is that no matter whether the comprehensive influence of neighbors in network is considered or not, initially, with the increase of $\beta_{0}$, the increased speed of $R$ shows that BA is the largest, Facebook is the second, and
WS network is the last. However, the final rumor size $R$ is $\mathrm{BA}>\mathrm{WS}>$ Facebook in the end. Besides, it is also crucial to understand how the final rumor size $R$ changes when considering the comprehensive influence of neighbors. Different from the network without considering the comprehensive influence of neighbors, the growth trend of $R$ in the network with considering the neighbors' influence presents an S-shaped curve when $\beta_{0}$ increases, which is described in a more detailed way rather than rapid rising and rapid falling. Undeniably, the stage when the final size $R$ of the rumor grows rapidly is the entry point which can be easy to control. By analyzing the trend of $R$, the neighbors' influence proposed can provide government and relevant control personnel a better theoretical basis for locating the entry point to take rumor control measures.

4.2.2. Influence of Perception Mechanisms on Propagation Process. The perception mechanisms are mainly the state transition dominated by the individual's subjective psychological changes in the process of rumor propagation, which is reflected in the probability $\delta$ and $\lambda$ of turning from the spreader and the weakener to the corrector.

As time goes by, the density of the spreader and the stifler changes with the spontaneous control rate $\delta$ as shown in Figure 7. In general, the larger $\delta$ is, the smaller the density of 


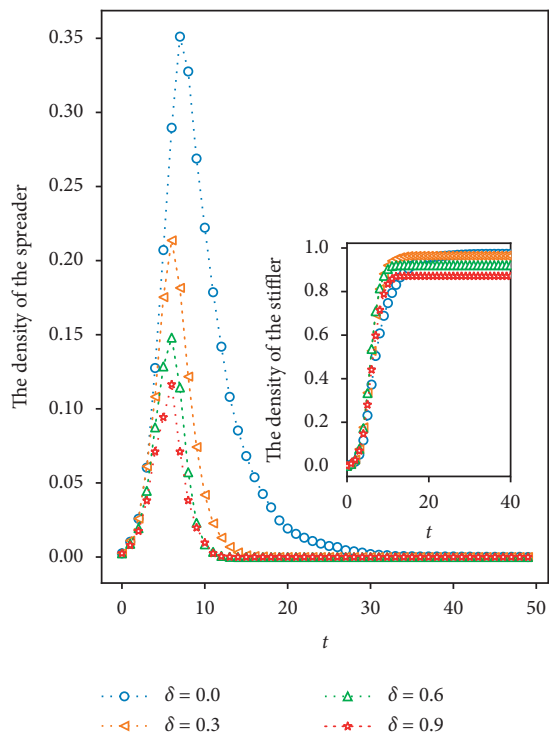

(a)

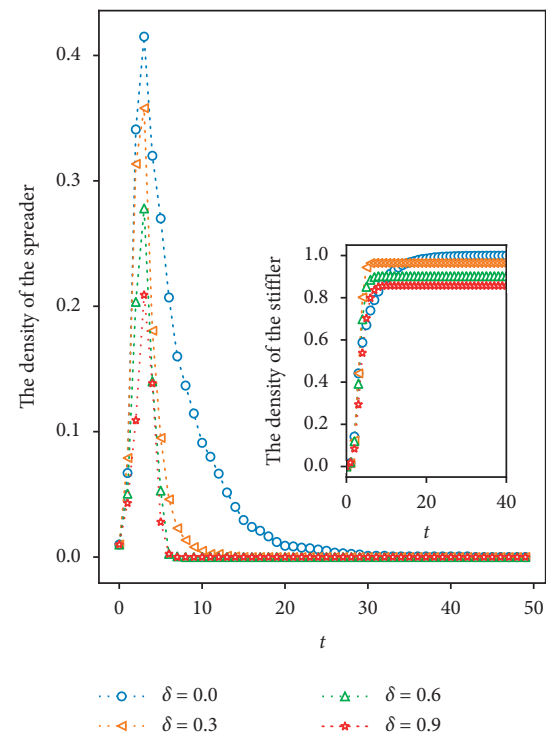

(b)

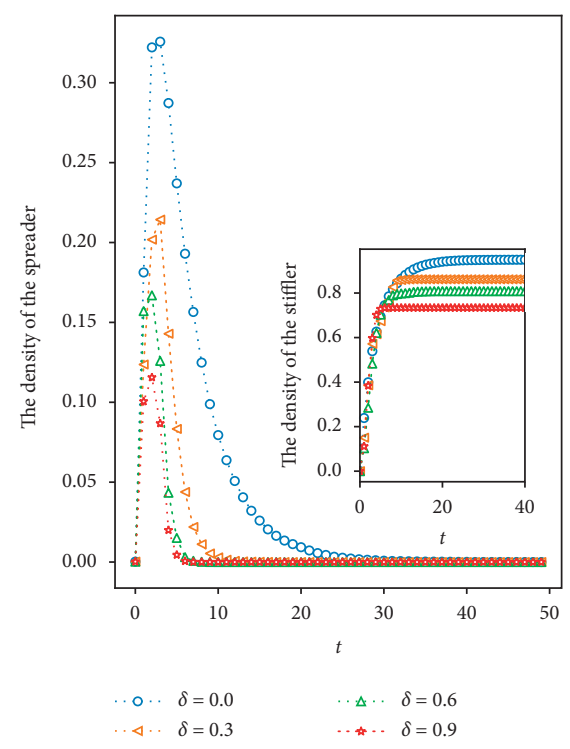

(c)

FIGURE 7: The density of the spreader with different $\delta$ on (a) WS network, (b) BA network, and (c) Facebook. The parameters are $\alpha=0.2$, $\beta_{0}=0.6, f=0.6, \eta=1-\beta-\alpha, \theta=0.1, r=0.1$, and $\lambda=0.05$.

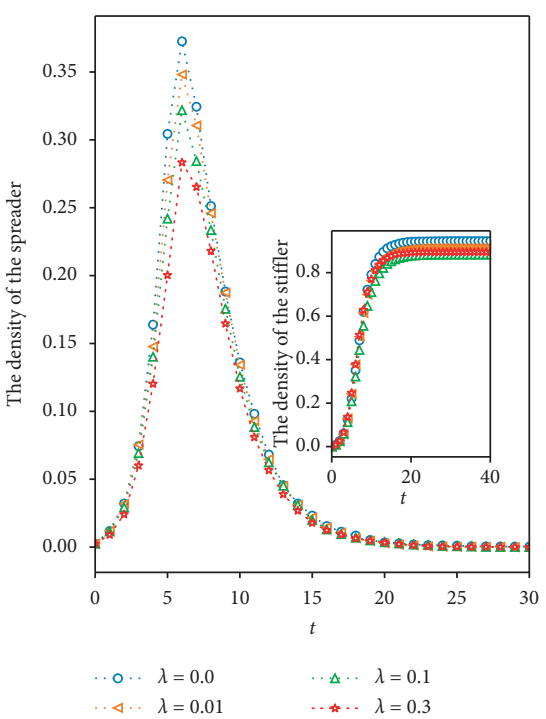

(a)

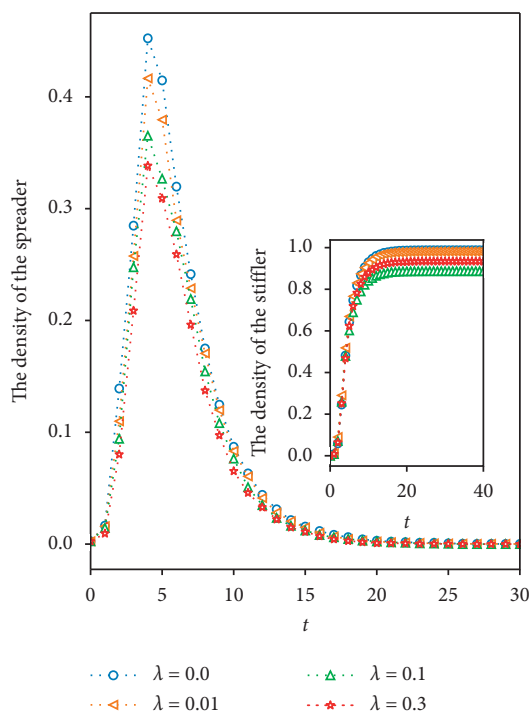

(b)

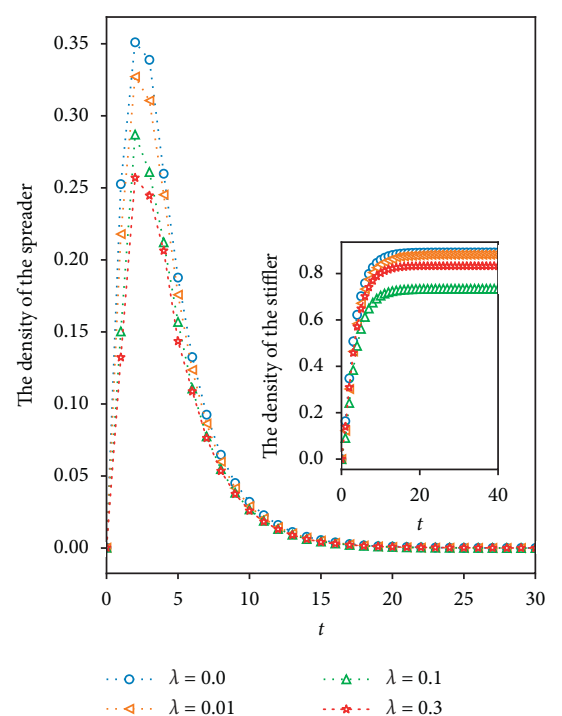

(c)

FIGURE 8: The density of spreader with different $\lambda$ on (a) WS network, (b) BA network, and (c) Facebook. The parameters are $\alpha=0.2$, $\beta_{0}=0.6, f=0.6, \eta=1-\beta-\alpha, \theta=0.1, r=0.1$, and $\delta=0.2$.

the spreader and the termination time of rumor are, which explains that the spreader's perception of life and the conversion to the corrector have a great influence on the individual's subjective choice in the face of rumor. Similarly, when comparing the final rumor size under different $\delta$, that is, the density of the stifler when $t \longrightarrow \infty$, the larger $\delta$ is, the smaller $R$ is. Furthermore outstanding is that, in WS and BA networks, $R$ has a similar interval change with the increase of $\delta$, while Facebook network has a smaller $R$. Therefore, it is necessary to call on the corrector who is transferred from the spreader for spreading his/her own perception experience actively to the friends around him/her, which is helpful for them to recognize the true face of the rumor.

Figure 8 shows the density trends of the spreader and the stifler with different $\lambda$ over time. Compared with Figures 8(a)$8(c)$, it is obvious that the trends of density change are similar under different networks. From the figures, the density of the spreader decreases with the increase of $\lambda$. Correspondingly, because $\lambda$ is the probability that the perception mechanism causes the weakener to be the corrector, there are more and 
more correctors in the system with the increase of $\lambda$. When $\lambda$ increases to a certain extent, the components of stiflers will be only correctors before the end of rumor propagation, and then more and more individuals in the system will be infected to correctors at the end of rumor propagation. Therefore, it explains that the density of the stifler in the figures shows an inverse growth trend with the increase of $\lambda$; that is, the density of the stifler when $\lambda=0.3$ is greater than that when $\lambda=0.1$. In life, weakener is a negative role of life attitude, and the actual probability of the transformation from weakener to corrector is relatively low in general. Due to the fact that the perception mechanism reflects a kind of life phenomenon, it cannot be ignored. The existence of weakeners with high fact transmission rate $\lambda$ in the system can effectively inhibit the spread of rumor.

\section{Conclusions}

In this paper, the C-SIW rumor propagation model with variable propagation rate and perception mechanism on social networks is proposed to explore the intrinsic motivation of state transformation. First of all, this paper employs a function to express the propagation rate, in which the spreader with high reputation can promote the spread of rumor. Besides, the variable propagation rate takes into account the comprehensive state of the neighbors contacted by individual, which further positions the stage of rapid growth of rumor propagation. In view of the possible influence of life on individuals, the paper also considers the perception mechanism. Experiments show that individuals with high perception probability including spontaneous control rate $\delta$ and fact transmission rate $\lambda$ can facilitate the reduction of rumor influence.

The paper proposes a theoretical model and verifies it by experiments in WS network, BA network, and Facebook. In actual life, when another event like rumor occurs, it may cause a review of the previous event. At this point, the constant parameter value of perception mechanism is not applicable. Therefore, the future work will explore the impact of the subsequent event on propagation process, no matter whether the subsequent event occurs during or after rumor propagation.

\section{Data Availability}

The WS and BA data used to support the findings of this study are included within the article, and the Facebook data used to support the findings of this study are available in the Stanford large network data collection website (http:// snap.stanford.edu/data/ego-Facebook.html).

\section{Conflicts of Interest}

The authors declare that there are no conflicts of interest regarding the publication of this paper.

\section{Acknowledgments}

This work was supported by the National Natural Science Foundation of China under Grant nos. 61502281 and 71772107.

\section{References}

[1] C. Li, L. Zhao, Q. Zeng, H. Duan, and Z. Zhao, "Learner2Vecbased learner community evolution analysis-a case study involving student card data," IEEE Access, vol. 7, pp. 2741627425, 2019.

[2] S. Gao, M. Zhou, Y. Wang et al., "Dendritic neuron model with effective learning algorithms for classification, approximation, and prediction," IEEE Transactions on Neural Networks \& Learning Systems, vol. 30, no. 2, pp. 601-614, 2018.

[3] D. J. Daley and D. G. Kendall, "Stochastic rumours," IMA Journal of Applied Mathematics, vol. 1, no. 1, pp. 42-55, 1965.

[4] D. P. Maki and M. Thompson, Mathematical Models and Applications, with Emphasis on the Social, Life, and Management Sciences, Prentice-Hall, Englewood Cliffs, NJ, USA, 1973.

[5] A. Sudbury, "The proportion of the population never hearing a rumour," Journal of Applied Probability, vol. 22, no. 2, pp. 443-446, 1985.

[6] Y. Moreno, M. Nekovee, and A. F. Pacheco, "Dynamics of rumor spreading in complex networks," Physical Review E, vol. 69, no. 6, Article ID 066130, 2004.

[7] M. Nekovee, Y. Moreno, G. Bianconi, and M. Marsili, "Theory of rumour spreading in complex social networks," Physica A: Statistical Mechanics and Its Applications, vol. 374, no. 1, pp. 457-470, 2007.

[8] Y.-Q. Wang, X.-Y. Yang, Y.-L. Han, and X.-A. Wang, "Rumor spreading model with trust mechanism in complex social networks," Communications in Theoretical Physics, vol. 59, no. 4, pp. 510-516, 2013.

[9] L.-L. Xia, G.-P. Jiang, B. Song, and Y.-R. Song, "Rumor spreading model considering hesitating mechanism in complex social networks," Physica A: Statistical Mechanics and Its Applications, vol. 437, pp. 295-303, 2015.

[10] L. A. Huo and Y. Y. Cheng, "Dynamical analysis of an IWSR rumor spreading model with considering the self-growth mechanism and indiscernible degree," Physica A, vol. 536, Article ID 120940, 2019.

[11] L. J. Zhao, J. J. Wang, and R. B. Huang, "Immunization against the spread of rumors in homogenous networks," PLoS One, vol. 10, no. 5, Article ID e0124978, 2015.

[12] J. Huang and X. Jin, "Preventing rumor spreading on smallworld networks," Journal of Systems Science and Complexity, vol. 24, no. 3, pp. 449-456, 2011.

[13] D. Kempe, J. M. Kleinberg, and E. Tardos, "Maximizing the spread of influence through a social network," in Proceedings of the 2003 ACM SIGKDD International Conference on Knowledge Discovery and Data Mining, pp. 137-146, Washington, DC, USA, 2003.

[14] J. Li, T. Sellis, J. S. Culpepper, Z. He, C. Liu, and J. Wang, "Geo-social influence spanning maximization," IEEE Transactions on Knowledge and Data Engineering, vol. 29, no. 8, pp. 1653-1666, 2017.

[15] J. Li, X. Wang, K. Deng et al., "Discovering influential community over large social networks," in Proceedings of the 2017 IEEE International Conference on Data Engineering, pp. 871-882, San Diego, CA, USA, April 2017.

[16] J. Li, T. Cai, K. Deng et al., "Community-diversified influence maximization in social networks," Information Systems, vol. 92, pp. 1-12, 2020.

[17] Z. Zhao, C. Li, X. Zhang, F. Chiclana, and E. H. Viedma, “An incremental method to detect communities in dynamic evolving social networks," Knowledge-Based Systems, vol. 163, pp. 404-415, 2019. 
[18] N. A. H. Haldar, J. Li, M. Reynolds et al., "Location prediction in large-scale social networks: an in-depth benchmarking study," VLDB Journal, vol. 28, no. 5, pp. 623-648, 2019.

[19] J. Li, C. Liu, J. X. Yu, Y. Chen, T. Sellis, and J. S. Culpepper, "Personalized influential topic search via social network summarization," IEEE Transactions on Knowledge and Data Engineering, vol. 28, no. 7, pp. 1820-1834, 2016.

[20] V. Indu and S. M. Thampi, "A nature-inspired approach based on forest fire model for modeling rumor propagation in social networks," Journal of Network and Computer Applications, vol. 125, pp. 28-41, 2019.

[21] K. Afassinou, "Analysis of the impact of education rate on the rumor spreading mechanism," Physica A: Statistical Mechanics and Its Applications, vol. 414, pp. 43-52, 2014.

[22] C. Y. Sang and S. G. Liao, "Modeling and simulation of information dissemination model considering user's awareness behavior in mobile social networks," Physica A, vol. 537, Article ID 122639, 2020.

[23] Y. Tian and X. J. Ding, "Rumor spreading model with considering debunking behavior in emergencies," Physica A, vol. 363, Article ID 124599, 2019.

[24] S. Ji, W. Yang, S. Guo, D. K. W. Chiu, C. Zhang, and X. Yuan, "Asymmetric response aggregation heuristics for rating prediction and recommendation," Applied Intelligence, vol. 50, no. 5, pp. 1416-1436, 2020.

[25] D. J. Watts and S. H. Strogatz, "Collective dynamics of "smallworld” networks," Nature, vol. 393, no. 6684, pp. 440-442, 1998.

[26] A.-L. Barabási and R. Albert, "Emergence of scaling in random networks," Science, vol. 286, no. 5439, pp. 509-512, 1999.

[27] J. Mcauley and J. Leskovec, "Learning to discover social circles in ego networks," Advances in Neural Information Processing Systems, vol. 1, pp. 539-547, 2012.

[28] H. Zhu, W. Hu, J. Ma et al., "Study on influence of community structure on opinion topic propagation of micro-blog," Journal of System Simulation, vol. 28, no. 7, 2016. 